\title{
THE
}

4-22-2003

\section{Taming the Rugged Landscape: Techniques for the Production, Reordering, and Stabilization of Selected Cluster Inherent Structures}

Dubravko Sabo

J. D. Doll

David L. Freeman

University of Rhode Island, dfreeman@uri.edu

Follow this and additional works at: https://digitalcommons.uri.edu/chm_facpubs

Terms of Use

All rights reserved under copyright.

\section{Citation/Publisher Attribution}

Sabo, D., Doll, J. D., \& Freeman, D. L. (2003). Taming the Rugged Landscape: Techniques for the Production, Reordering, and Stabilization of Selected Cluster Inherent Structures. Journal of Chemical Physics, 118(16), 7321-7328. doi: 10.1063/1.1562621

Available at: http://dx.doi.org/10.1063/1.1562621

This Article is brought to you for free and open access by the Chemistry at DigitalCommons@URI. It has been accepted for inclusion in Chemistry Faculty Publications by an authorized administrator of DigitalCommons@URI. For more information, please contact digitalcommons-group@uri.edu. 


\title{
Taming the rugged landscape: Techniques for the production, reordering, and stabilization of selected cluster inherent structures
}

\author{
Dubravko Sabo a) and J. D. Doll \\ Department of Chemistry, Brown University, Providence, Rhode Island 02912 \\ David L. Freeman \\ Department of Chemistry, University of Rhode Island, Kingston, Rhode Island 02881
}

(Received 2 December 2002; accepted 31 January 2003)

\begin{abstract}
We report our studies of the potential energy surface (PES) of selected binary Lennard-Jones clusters. The effect of adding selected impurity atoms to a homogeneous cluster is explored. Inherent structures and transition states are found by combination of conjugate gradient and eigenvector-following methods while the topography of the PES is mapped with the help of a disconnectivity analysis. We show that we can controllably induce new structures as well as reorder and stabilize existing structures that are characteristic of higher-lying minima. (C) 2003 American Institute of Physics. [DOI: 10.1063/1.1562621]
\end{abstract}

\section{INTRODUCTION}

The minimization/optimization problem is one of the more ubiquitous and challenging in computational science. ${ }^{1}$ Central to researchers in the physical sciences and engineering, this problem is also of primary importance to social, biological, and economics investigators.

Driven in large measure by such widespread interest, there has been appreciable progress on the minimization problem. Especially notable have been algorithmic advances in the form of annealing and stochastic relaxation approaches ${ }^{1-6}$ as well as basin-hopping techniques. ${ }^{7,8}$ In both classical and quantum form, these methods offer valuable, complementary alternatives to traditional, gradient or pseudogradient approaches. ${ }^{1}$

In addition to algorithmic developments relevant to the minimization problem, there have also been notable advances in the tools to classify and analyze the topography of the underlying objective functions. In chemical applications, the principal focus of the remainder of our discussion, the objective function of interest is typically a specified potential or free energy surface. Following Stillinger and Weber, ${ }^{9,10}$ it is useful to perform an "inherent structure" decomposition of the associated configuration space by employing the minima (local and global) of this surface. These inherent structures, their relative orderings, and their connectivity provide important information concerning the structure, function, and dynamics of the associated physical system. Disconnectivity analysis introduced by Czerminski and Elber, ${ }^{11}$ discussed by Becker and Karplus, ${ }^{12}$ and developed by, among others, Wales, Doye, and Miller ${ }^{13-15}$ has proved especially valuable with respect to these latter tasks.

As evidenced by the development of classical and quantum annealing methods, there is an important interplay between minimization and the Monte Carlo sampling problem. Both applications, for example, are concerned with overcom-

${ }^{a)}$ Electronic mail: dubravko_sabo@brown.edu ing barriers that inhibit the interconversion or isomerization of the various inherent structures of the problem. Consequently, developments in one field contain implications for developments in the other. Advances in rare event sampling methods, such as J-walking ${ }^{16,17}$ and parallel tempering ${ }^{18-21}$ methods, thus contain implications for the minimization problem.

The field of atomic and molecular clusters has been and continues to be an important test bed for the development and application of minimization and analysis methods. Utilizing the above-outlined methods, researchers have produced a relatively coherent picture of the relationship between the nature of underlying potential energy surface (PES) and the physical properties of the associated systems. For example, from the studies by Berry et al., ${ }^{22-25}$ the single component studies of Wales et al., ${ }^{13-15}$ the mixed LennardJones (LJ) cluster studies of Jordan et al. ${ }^{26}$ as well as the research of others, ${ }^{27-30}$ we have begun to understand the nature of systems for which the lowest inherent structures can or cannot be readily located.

In present paper we would like to build upon advances in the minimization problem by effectively turning the logic "upside down." That is, instead of asking what we have to do in order to locate or sample the global minimum of a specified potential energy surface, we wish instead to ask how we might go about controllably inducing new structures as well as reordering and stabilizing existing structures that are characteristic of higher-lying local minima. Basically, we seek to utilize what we have learned about what it takes to avoid local minima to instead controllably produce them.

In principle, one can envision efforts involving both thermodynamic and kinetic approaches. In the present work we shall focus principally on the thermodynamic issues. Furthermore, we shall limit the discussion in the present work to applications involving clusters. As discussed elsewhere, ${ }^{31}$ clusters are of appreciable technological importance, are valuable as prototypes for the study of the properties of extended systems, illuminate issues related to the size- 
dependence of selected physical properties, and provide valuable test beds for the development and application of emerging computational techniques. This combination of formal, computational, and technological interest has produced a vast and growing cluster literature. ${ }^{31-33}$

The remainder of the paper is organized as follows: In Sec. II we outline the computational details of the present study. We discuss the methods we use to determine the inherent structures and transition states of a specified cluster's PES. Using these methods, we examine specific results for two prototype systems in Sec. III. These particular results are designed to demonstrate "proof of principle" with respect to the basic objectives of the present study for selected systems. Finally, in Sec. IV we summarize our results and speculate about likely future research directions.

\section{COMPUTATIONAL DETAILS}

The present section describes the computational details of our investigations involving binary clusters of the form $X_{n} Y_{m}$. Our overall interest will be to explore the extent to which we can utilize the "adatoms" (i.e., the $Y$ system) to induce, reorder, and stabilize selected inherent structures in the "core" $X$ system. While one can easily imagine applications involving both more and more complex components, we feel these relatively simple, two-component clusters are a convenient starting point for an initial study of the issues we raise.

We shall assume in what follows that the total potential energy is composed of a pairwise sum of Lennard-Jones interactions. Specifically, we assume that the total potential energy, $V_{\text {tot }}$, for an $N$-particle system is given by

$$
V_{\mathrm{tot}}=\sum_{i<j}^{N} v_{i j}\left(r_{i j}\right)
$$

where the pair interaction as a function of the distance between particles $i$ and $j, r_{i j}$, is given by

$$
v_{i j}\left(r_{i j}\right)=4 \epsilon_{i j}\left[\left(\frac{\sigma_{i j}}{r_{i j}}\right)^{12}-\left(\frac{\sigma_{i j}}{r_{i j}}\right)^{6}\right] .
$$

In Eq. (2) the constants $\epsilon_{i j}$ and $\sigma_{i j}$ are the energy and lengthscale parameters for the interaction of particles $i$ and $j$.

For a two-component system, we must specify both the "like" $(X-X, Y-Y)$ as well as the "mixed" $(X-Y)$ interactions. With an eye toward studying trends in the results as opposed to results for particular physical systems, it is convenient to reduce the number of free parameters. To do so, we shall assume in the present study that the "mixed" Lennard-Jones values are obtained from the "like" LennardJones parameters via usual combination rules ${ }^{34}$

$$
\begin{aligned}
& \sigma_{X Y}=\frac{1}{2}\left(\sigma_{X X}+\sigma_{Y Y}\right), \\
& \epsilon_{X Y}=\sqrt{\epsilon_{X X} \epsilon_{Y Y}} .
\end{aligned}
$$

Furthermore, we note that with the mixed Lennard-Jones parameters specified as in Eqs. (3) and (4), the inherent structure topography of the "reduced" potential energy surface of the binary system (i.e. $V_{\text {tot }} / \epsilon_{X X}$ ) is a function of only two parameters, $(\sigma, \epsilon)$, the ratios of the corresponding adatom/ core length and energy parameters,

$$
\begin{aligned}
& \sigma=\sigma_{Y Y} / \sigma_{X X}, \\
& \epsilon=\epsilon_{Y Y} / \epsilon_{X X} .
\end{aligned}
$$

If necessary for a discussion of a specific physical system, the absolute bond lengths, energies, activation energies, etc., can be obtained from the corresponding "reduced" results by a simple rescaling with the appropriate core-system Lennard-Jones parameters.

The computational task in our study is thus one of exploring and characterizing the (reduced) potential energy surface of our binary cluster systems as a function of the number of (core, adatom) particles, $(n, m)$, and for given $(\sigma, \epsilon)$ ratios. In typical applications the lowest $N_{\text {IS }}$ inherent structures and the associated disconnectivity graphs are determined. For the applications reported here, $N_{\text {IS }}$ is generally of the order of a few hundred (thousand) or less. Depending on the size of the cluster, inherent structures are found either via conjugate gradient methods starting from randomly chosen initial configurations, or by more systematic surface exploration methods such as those outlined by Wales and co-workers ${ }^{14}$ and by Jordan et al. ${ }^{35}$ In all cases, the inherent structures that are located are confirmed to be stable minima via a standard Hessian analysis. To reduce the chance we miss particular local or global minima, we monitor the number of times individual inherent structures are found and demand that each of the $N_{\text {IS }}$ inherent structures be located a minimum number of times (at least 10) before we terminate our search. Once we are satisfied we have located the relevant inherent structures, transition states linking these stable minima are obtained using the eigenvector following methods outlined by Cerjan and Miller ${ }^{36}$ and further developed by Simons et al., ${ }^{37-39}$ Jordan et al., ${ }^{35}$ and Wales. ${ }^{40}$ Finally, with the requisite inherent structures and barriers in hand, we perform a disconnectivity analysis using methods outlined by Czerminski and Elber, ${ }^{11}$ Becker and Karplus, ${ }^{12}$ and Miller et al. $^{13}$

\section{NUMERICAL RESULTS}

In the present section, we wish to illustrate the general themes we introduced in Sec. I. We do so by demonstrating that we can accomplish three basic objectives. Specifically, we show that by adding selected "impurity" atoms to bare "core" systems, we can:

(1) induce new "core structures,"

(2) reorder the energies of existing core inherent structures, and

(3) stabilize selected inherent structures by controlling the activation energies that determine their isomerization kinetics.

For purposes of illustration, we shall examine numerical results for a few, simple Lennard-Jones systems involving five and seven core atoms, systems well-known from previous studies to have one and four energetically distinct inher- 


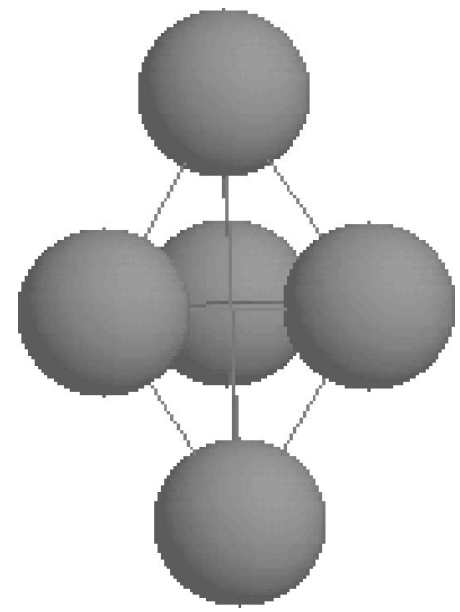

FIG. 1. The only stable inherent structure for $X_{5}$ LJ cluster. Its energy (in units of the LJ well depth) is -9.104 .

ent structures, respectively. The inherent structures and their associated energies for these core systems are illustrated in Figs. 1 and 2.

We first consider mixed clusters of the generic type $X_{5} Y_{2}$. Here two impurity $Y$ atoms are added to the parent, five-atom $X$ core. We have chosen this system because it builds upon the very simple five-atom core, a system that has only a single inherent structure, and because the total system has a total of seven atoms, a magic number for icosahedral growth in homogeneous systems. Using the techniques of Sec. II, we then determine the lowest several inherent structures for a range of $(\sigma, \epsilon)$ [c.f. Eqs. (5) and (6)]. As can be seen from Fig. 3, the total potential energy [Eq. (1)] of the

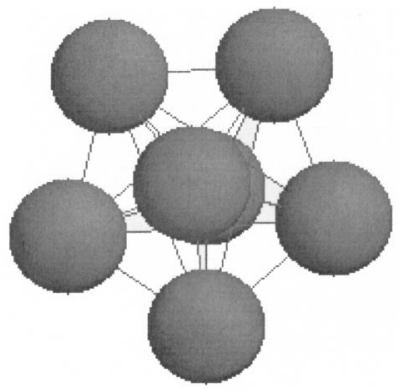

(a)

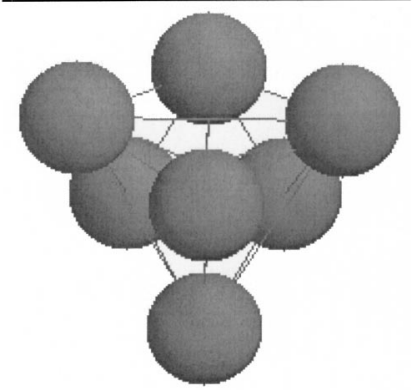

(c)

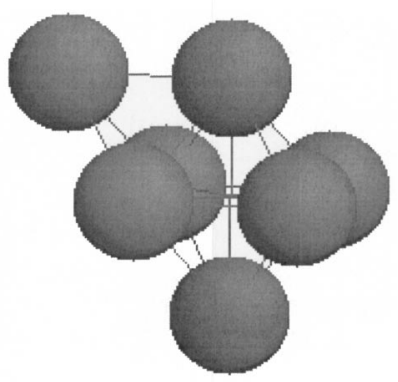

(b)

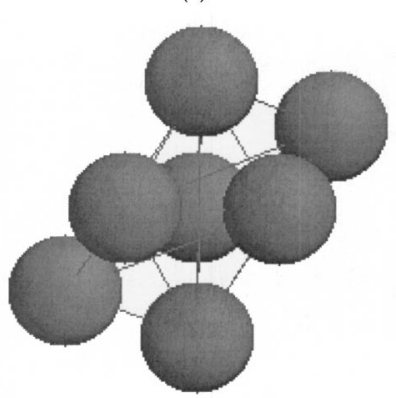

(d)
FIG. 2. The four, energetically distinct, stable inherent structures for $X_{7} \mathrm{LJ}$ cluster. The energies (in units of the LJ well depth) are: (a) -16.505 , (b) -15.935 , (c) -15.593 , (d) -15.533 .

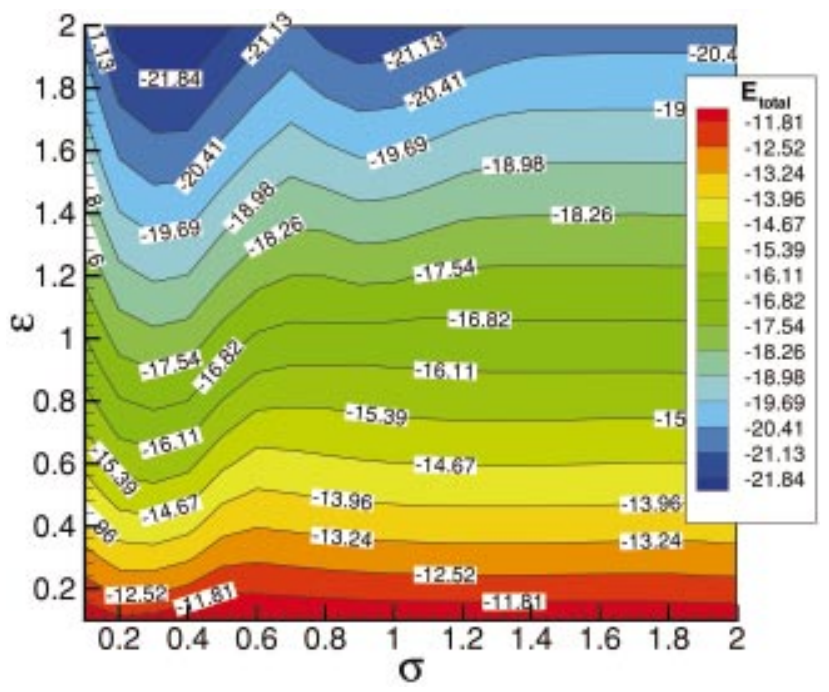

FIG. 3. (Color) $E_{\text {tot }}(\sigma, \epsilon)$ [c.f. Eqs. (1), (5), and (6)] for the $X_{5} Y_{2}$ system. Note the relative lack of structure in the $(\sigma, \epsilon)$ variation of the total cluster energy.

lowest inherent structure for the $X_{5} Y_{2}$ system shows no appreciable structure as a function of the $(\sigma, \epsilon)$ parameters.

On the other hand, we see in Fig. 4 that the core potential energy, defined as the potential energy of interaction for only the core $X$ atoms, of the minimum (total) energy cluster clearly breaks into extended regions, each corresponding to a well-defined core structure. The reader should notice that each region in Fig. 4 contains the same "kind" of core structure but their core energies are slightly different. We have chosen a single "average" core energy value to represent all energies in the corresponding domain for plotting convenience.

The distinct core structures, shown in Fig. 4, have been identified by examining their core energies $\left(E_{\text {core }}\right)$ and their

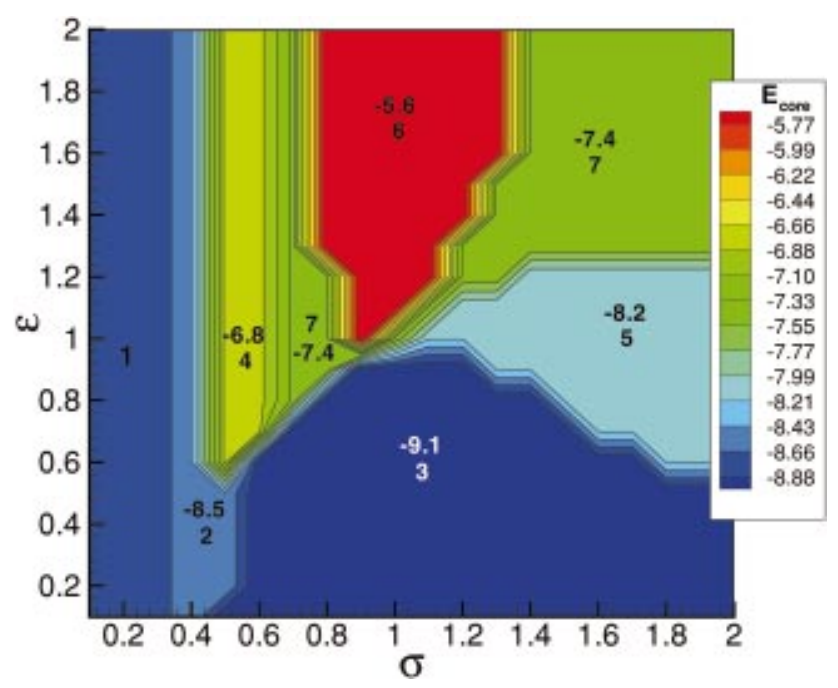

FIG. 4. (Color) $E_{\text {core }}(\sigma, \epsilon)$ for the $X_{5} Y_{2}$ system. Here the "core" energy is defined as that portion of the potential energy arising from only the corecore atom interactions. Unlike the total energy, the $(\sigma, \epsilon)$ variation of the core cluster energy exhibits relatively well-defined regions. The labels of each of these regions correspond to the distinct core structures shown in Fig. 5. 


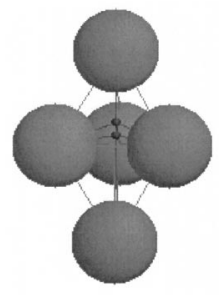

(5.1)
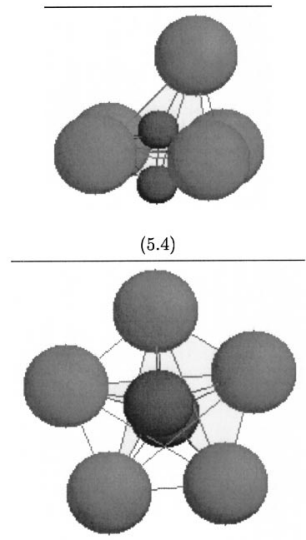

(5.6)

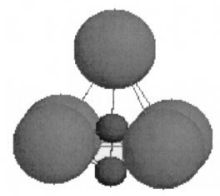

$(5.2)$

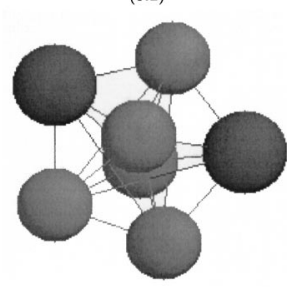

(5.5)

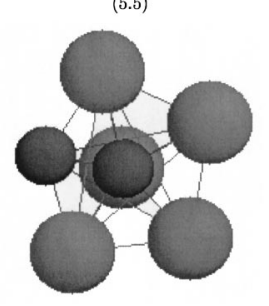

(5.7)

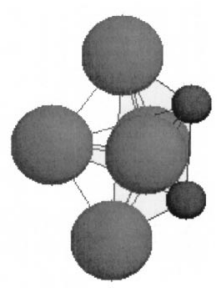

$(5.3)$
FIG. 5. Plots of $X_{5} Y_{2}$ structures for selected $(\sigma, \epsilon)$ values. The decimal number for each figure denotes the corresponding $(\sigma, \epsilon)$ domain in Fig. 4.

principal moments of inertia. For each structure a triplet of values $\left(E_{\text {core }}, I_{2}, I_{3}\right)$ has been associated, where $I_{2}$ and $I_{3}$ are the moments of inertia about the principal axes 2 and 3 , respectively. We have defined $I_{2}$ and $I_{3}$ in the following way: $I_{2}=I_{2}^{\prime} / I_{1}^{\prime}, I_{3}=I_{3}^{\prime} / I_{1}^{\prime}$ where $I_{1}^{\prime}, I_{2}^{\prime}$, and $I_{3}^{\prime}$ are the principal moments of inertia obtained by diagonalizing the inertia tensor of the system. If the triplet of values has not been sufficient to identify a core structure then we have examined the structure visually.

Selected cluster structures illustrating the core arrangements corresponding to various $(\sigma, \epsilon)$ values are shown in Fig. 5. We see from Figs. 4 and 5 that the $X_{5} Y_{2}$ cluster exhibits core $X$-atom structures that include trigonal bipyramidal, planar, and square pyramidal core geometries. Of these, only the trigonal bipyramidal form is stable in the parent $X_{5}$ system. This illustrates that a suitable choice of the $(\sigma, \epsilon)$ parameters can controllably induce core geometries not present as stable minima in the bare cluster. For example, the square pyramid core structure, seen in Fig. 5.2 as a stable system, corresponds to a transition state in the bare $X_{5}$ cluster.

Figure 6 represents the $X_{5} Y_{2}$ cluster at four points in Fig. 4 defined by the $(\sigma, \epsilon)$ coordinates $(0.4,0.5),(0.4,1.0)$, $(0.4,1.5)$, and $(0.4,2.0)$. Here the pairs of coordinates correspond to (a), (b), (c) and (d) of Fig. 6, respectively. In other words, we keep value of $\sigma=0.4$ fixed, while increasing the value of $\epsilon$. Each disconnectivity graph shows all inherent structures available to the system for the given $(\sigma, \epsilon)$ values. The global minimum of each system is labeled by number 1 and contains as a recognizable component the square pyramid core structure (see Fig. 5.2). In Fig. 6(a) the square pyramid core structure is connected to two inherent structures, labeled 2 and 3 , by pathways whose energies do not exceed -13.8 (in units of $\epsilon_{X X}$ ). Since isomer 2 contains the same core structure (the square pyramid) as the global minimum the corresponding isomerization thus does not lead to a change in the core structure of the cluster. For present purposes, therefore, the barrier that connects them is not a "relevant" barrier. The relevant barriers are those that connect inherent structures that contain different core structures. The inherent structure 3 contains as the core structure a (distorted) trigonal bipyramid (see Fig. 5.3). Therefore, the isomerization barrier that connects the inherent structure 3 with global minimum is the lowest relevant isomerization barrier and its value is $\Delta E_{1,3}=0.986 \epsilon_{X X}$. Figures 6(b) and 6(c) show that increasing the value of $\epsilon$ increases isomerization barriers that connect inherent structure 1 (the square pyramid core structure) with inherent structure 2 (the distorted trigonal bipyramid core structure). Numerically, these barriers are $\Delta E_{1,2}=1.227 \epsilon_{X X}$ and $\Delta E_{1,2}=1.431 \epsilon_{X X}$, respectively. In Fig. 6(d) the square pyramid core structure is connected by an isomerization barrier of $\Delta E_{1,2}=1.647 \epsilon_{X X}$ with two (almost degenerate in energy) distorted trigonal bipyramid core structures. As illustrated in Fig. 6 and discussed earlier, the barriers that determine the isomerization kinetics of these newly induced structures are sensitive to the $(\sigma, \epsilon)$ values and can thus be at least partially controlled. These two simple results are specific demonstrations of goals (1) and (3) stated earlier.

As a second illustration, we consider mixed clusters of the type $X_{7} Y_{3}$. This system builds upon a parent, sevenatom, "magic number" system known to exhibit a set of four, energetically distinct inherent structures. The core inherent structures and associated energies for the stable $X_{7}$ inherent structures are presented in Fig. 2. Figure 7, a $(\sigma, \epsilon)$ contour plot of the core-atom potential energies of the lowest total energy $X_{7} Y_{3}$ clusters, again reveals the presence of definite "core-phases." As illustrated in Fig. 8, some of these regions correspond to various core structures present in the parent $X_{7}$ system while others correspond to new structures not seen in the original, single-component cluster. We can see from Figs. 7 to 9 that the impurity $Y$ atoms provide us with significant control over the relative ordering of the core energies of the parent $X_{7}$ system. Specifically, by choosing an appropriate range of $(\sigma, \epsilon)$ values, we can generate $X_{7} Y_{3}$ clusters in which the lowest (total) energy inherent structure can have core structures that are either pentagonal bipyramid, capped octahedral, or bicapped trigonal bipyramidal in nature. Moreover, since we can manipulate the isomerization barriers in these systems, we can at least partially stabilize clusters that exhibit selected core structures with respect to isomerization. This is illustrated in Fig. 9.

Figures 9(a)-9(d) represent the $X_{7} Y_{3}$ cluster at four points in Fig. 7 with $X_{7} Y_{3}(\sigma, \epsilon)$ coordinates $(0.4,0.5)$, $(0.4,1.0),(0.4,1.5)$, and $(0.4,2.0)$, respectively. The number of inherent structures available to the $X_{7} Y_{3}$ cluster varies from more than 800 in Fig. 9(a) to 400 in Fig. 9(d). Since we are primarily interested in energetically low-lying inherent structures we show only lowest 70 inherent structures. The global minimum of each system is labeled by number 1 and 


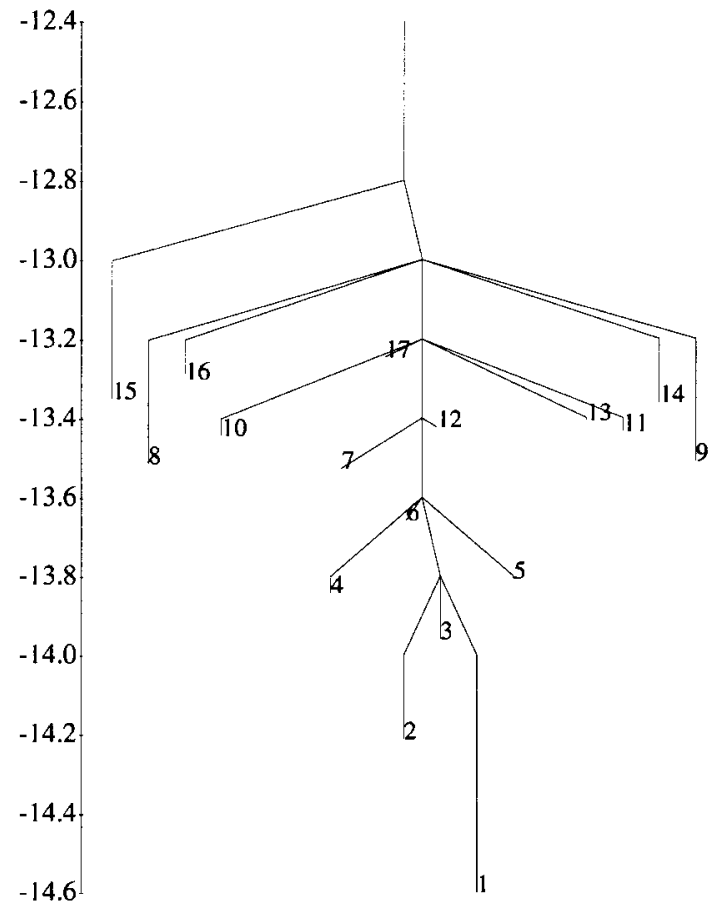

(a)

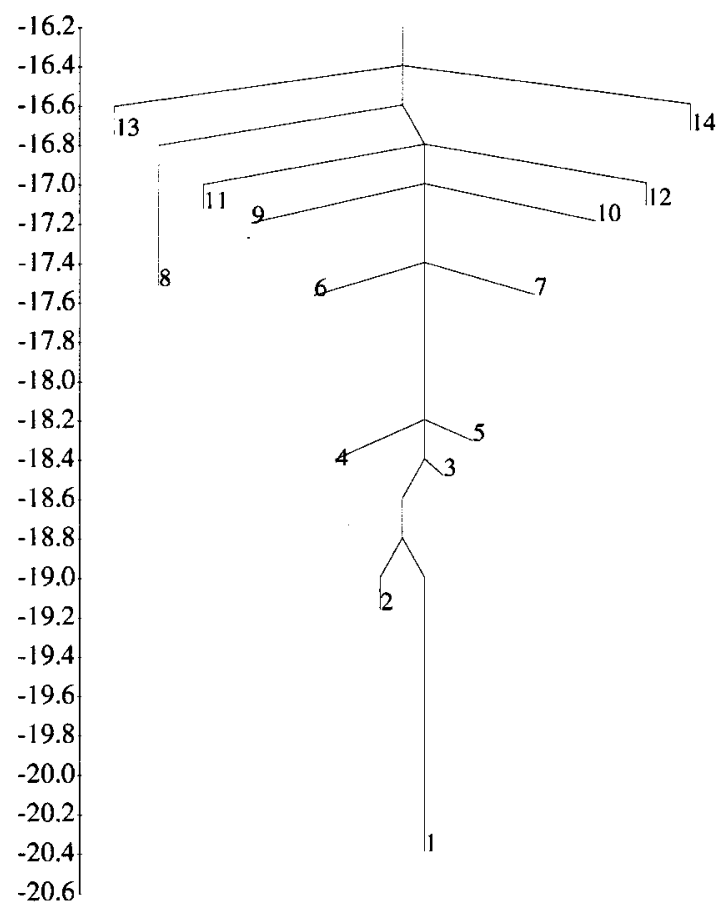

(c)

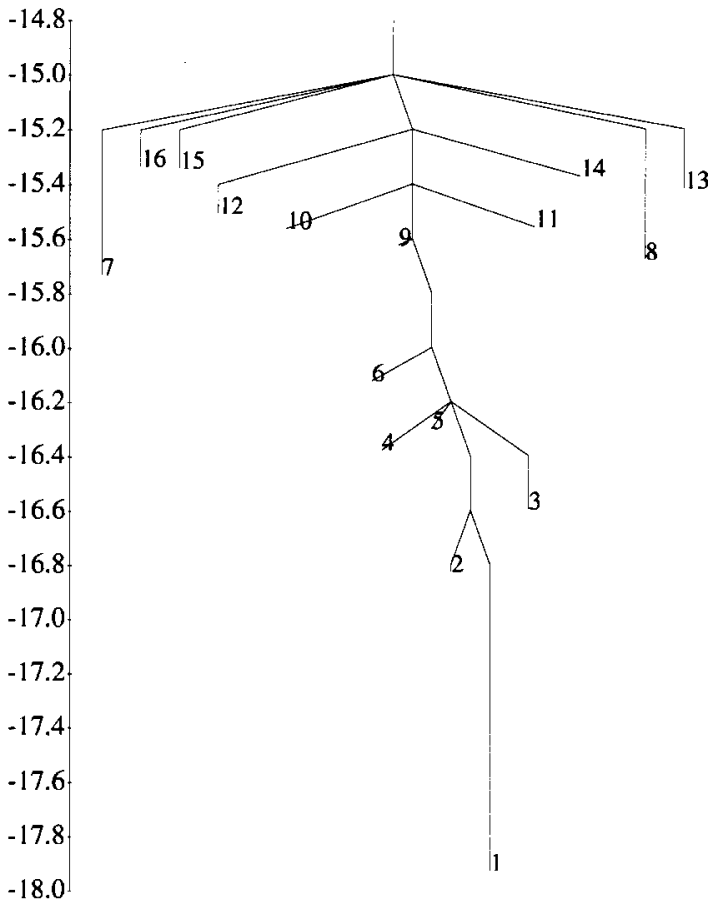

(b)

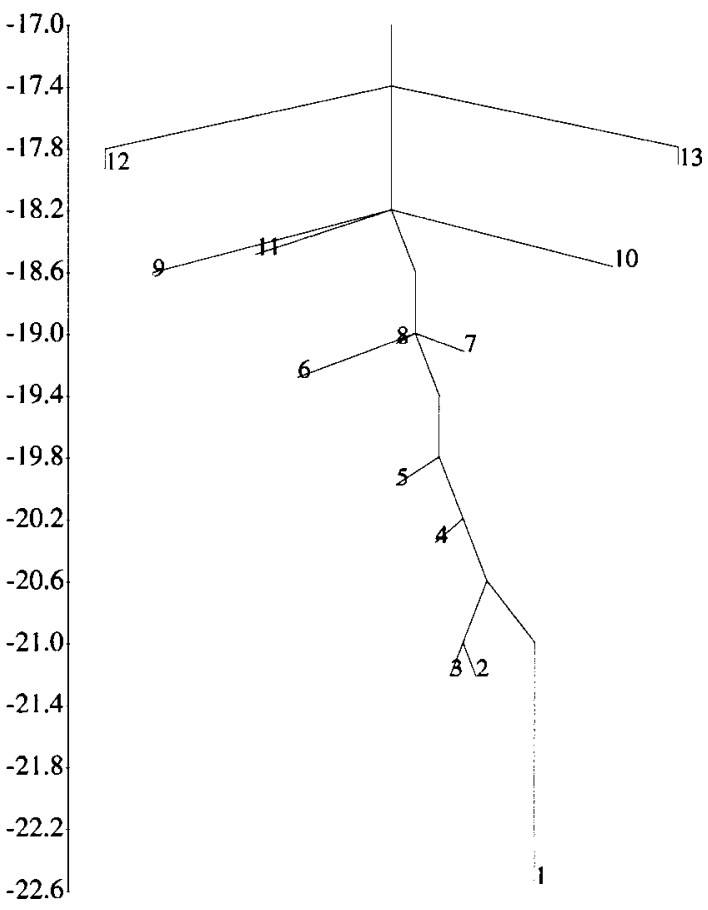

(d)

FIG. 6. Disconnectivity graph for $X_{5} Y_{2}(\sigma, \epsilon)$ values demonstrating that we can control barriers for the selected inherent structures. The energy scale is in units of $\epsilon_{X X}$. The $(\sigma, \epsilon)$ values for panels (a)-(d) are $(0.4,0.5),(0.4,1.0),(0.4,1.5)$, and $(0.4,2.0)$, respectively.

contains as a recognizable component the core structure shown in Fig. 8.5. We should mention that for a given range of $\sigma$ and $\epsilon$ values, $(\sigma, \epsilon) \in[0.1,2.0]$, we have not been able to find a global minimum that would contain as a recogniz- able component inherent structure 4 of the parent $X_{7}$ cluster [see Fig. 2(d)]. This is the reason why none of the domains in Fig. 7 is labeled by number 4. In Fig. 9(a) the global minimum, the core structure 5 (see Fig. 8.5), is linked to inherent 


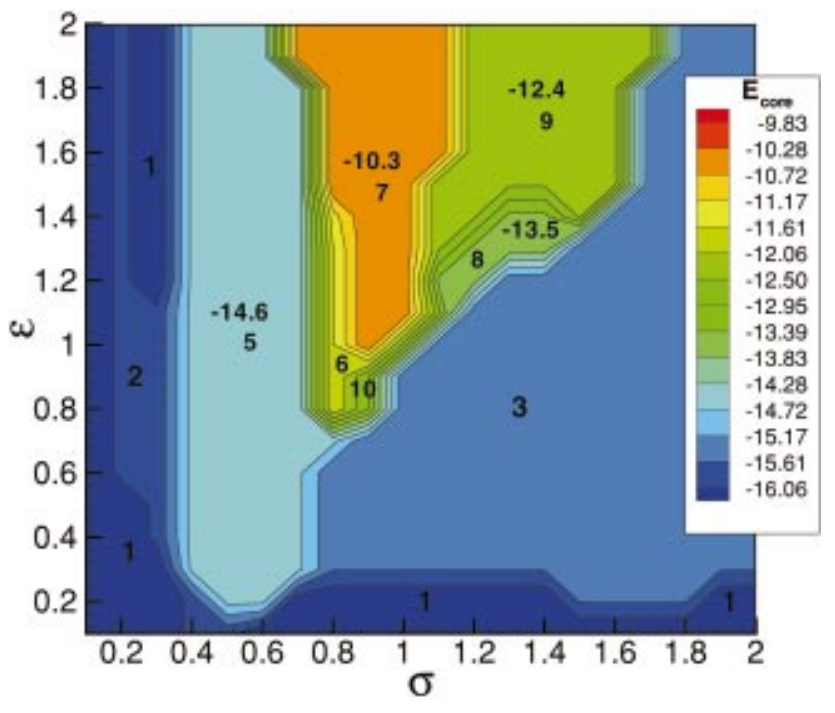

FIG. 7. (Color) $E_{\text {core }}(\sigma, \epsilon)$ for $X_{7} Y_{3}$. Format for the plot is the same as in Fig. 4.

structure 2 which contains the (distorted) capped octahedron core structure (see Fig. 8.2). The isomerization barrier between them is $\Delta E_{1,2}=0.494 \epsilon_{X X}$. Figures $9(\mathrm{~b})$ and $9(\mathrm{c})$ show that increasing the value of $\epsilon$ increases isomerization barriers, that connect inherent structure 1 (see core structure in Fig. 8.5) with inherent structures 3 and 2 (a distorted capped octahedron core structure), respectively. Numerically, these barriers are $\Delta E_{1,3}=0.975 \epsilon_{X X}$ and $\Delta E_{1,2}=1.136 \epsilon_{X X}$, respectively. The inherent structure 2 in Fig. 9(b) contains the same core structure as the global minimum and, therefore, has not been considered relevant for the isomerization [see the above-mentioned explanation for Fig. 6(a)]. In Fig. 6(d) the core structure 5 is connected to two, energetically almost degenerate, inherent structures labeled by 2 and 3, by pathways whose energies do not exceed -40.5 (in units of $\epsilon_{X X}$ ). Similar to the case of Fig. 6(a), isomer 2 contains the same core structure as the global minimum so the corresponding isomerization does not lead to a change in the core structure of the cluster. The barrier that links them is not a relevant barrier. The inherent structure 3 contains as the core structure a distorted capped octahedron. Therefore, the isomerization barrier which connects the inherent structure 3 with global minimum is the lowest relevant isomerization barrier and its value is $\Delta E_{1,3}=1.304 \epsilon_{X X}$.

\section{CONCLUSIONS}

In the present work, we have considered the general task of altering core cluster structures. We are, in effect, attempting to turn the logic of the minimization problem upside down. Rather than seeking the global minimum of complex potential energy surfaces, we are instead attempting to exploit what has been learned about the general minimization problem to controllably alter core cluster structures. Specifically, we are examining the extent to which we can induce new core geometries as well as reorder and stabilize existing, higher-lying, local core structures.

Our approach, in the present discussion, has been thermodynamic in nature. We have utilized selected adatoms to

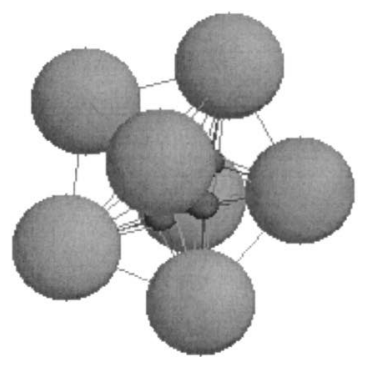

(8.1)

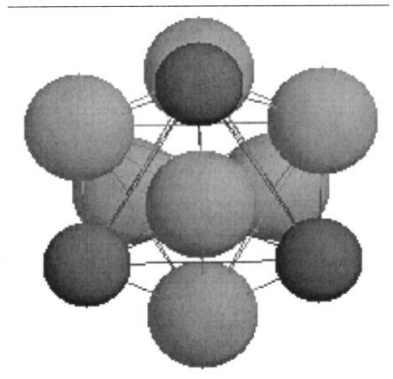

(8.3)

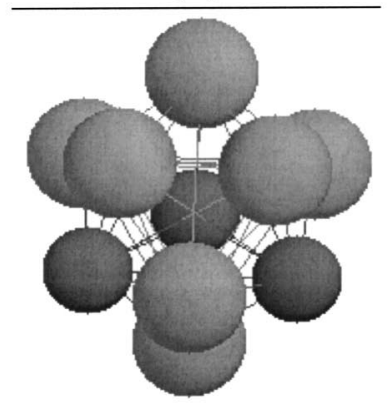

(8.6)

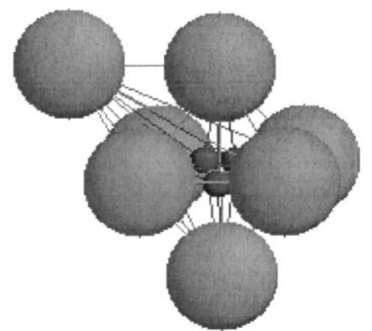

$(8.2)$

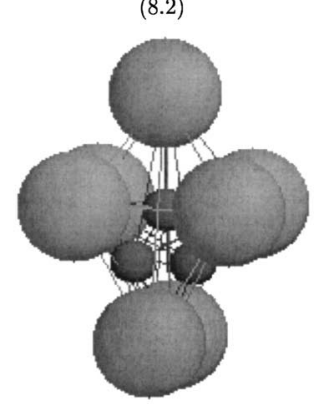

$(8.5)$

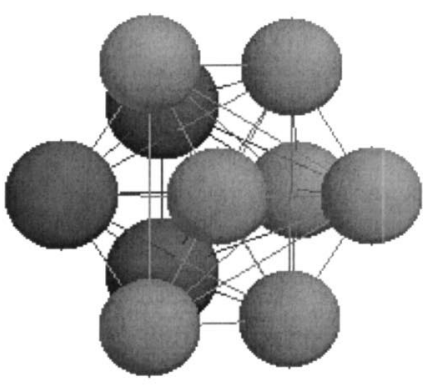

$(8.8)$
FIG. 8. Plots of selected $X_{7} Y_{3}$ structures for various $(\sigma, \epsilon)$ values identified in Fig. 7. The number of the structures correspond to the regions labeled in Fig. 7. Note that many of the core structures for these systems are not stable energy structures of the bare $X_{7}$ system.

effect our desired core cluster modifications. We have presented results for two simple binary cluster examples, the $X_{5} Y_{2}$ and $X_{7} Y_{3}$ systems, to validate our approach.

We speculate that there are at least two important directions for future theoretical development of the present ideas. One direction will be to explore the use of more complex adsorbates to achieve selected core cluster structures. One could, for example, imagine using "exterior" methods in which encapsulating agents of well-defined geometries were utilized to induce desired core structures. Alternatively, "interior" approaches in which complex objects, perhaps even previously engineered clusters, could be utilized as "seeds" or "templates" to produce a desired structure in the surrounding cluster (either globally or locally). Another important direction will be to explore the extent to which previously engineered cluster structures can be assembled using "cluster assembled materials" methods to produce larger scale, macroscopic structures. If this proves possible, it would seem to offer an important direction in the production of novel materials starting from synthetic precursors whose core structures and properties are highly varied and are under user control. 


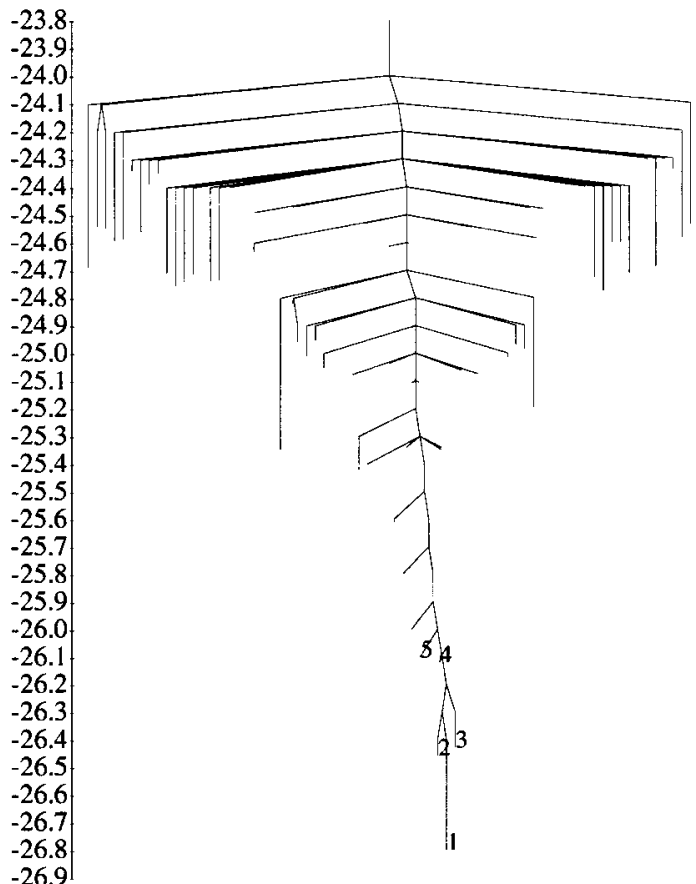

(a)

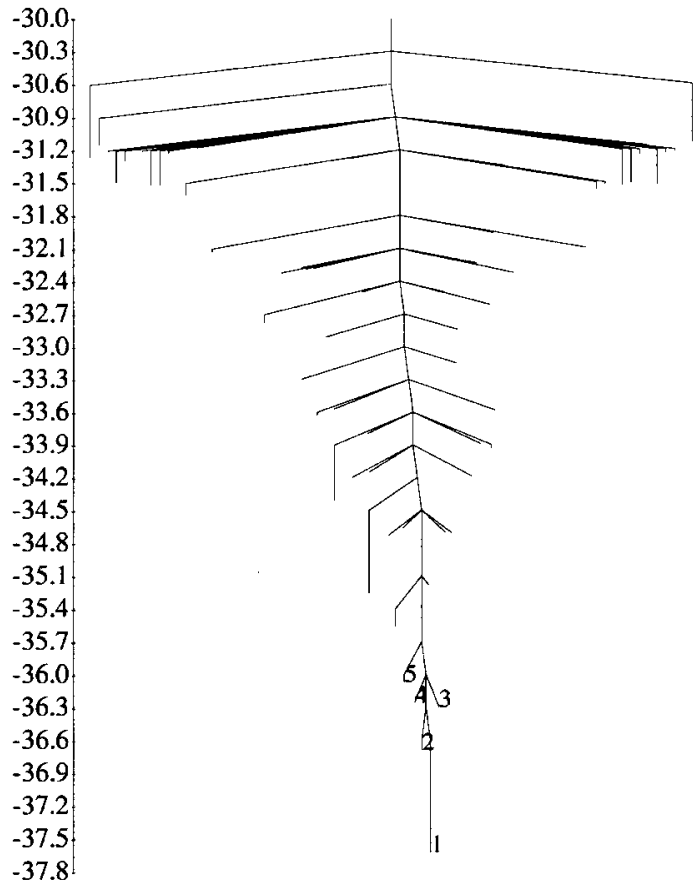

(c)

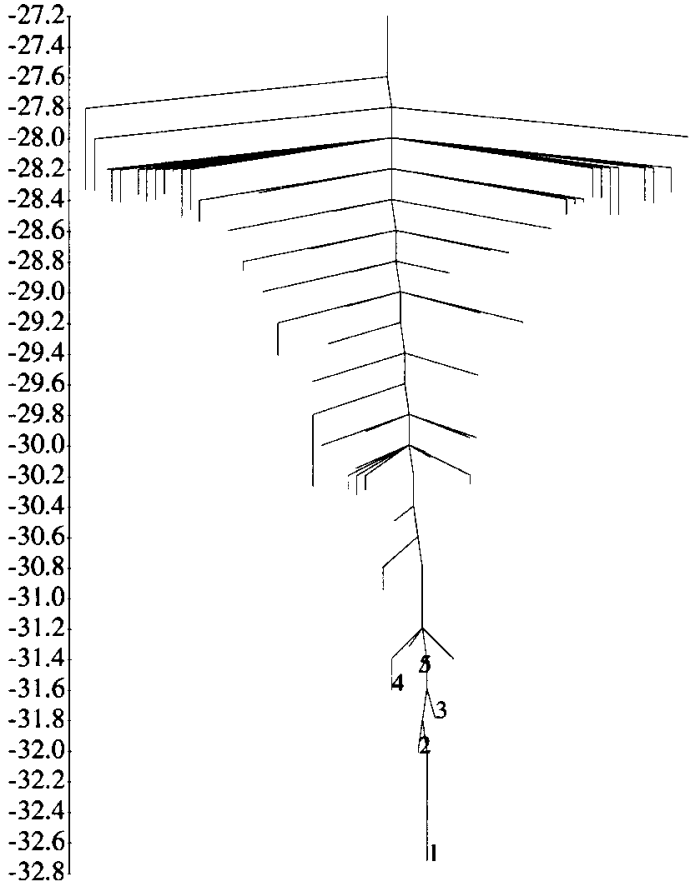

(b)

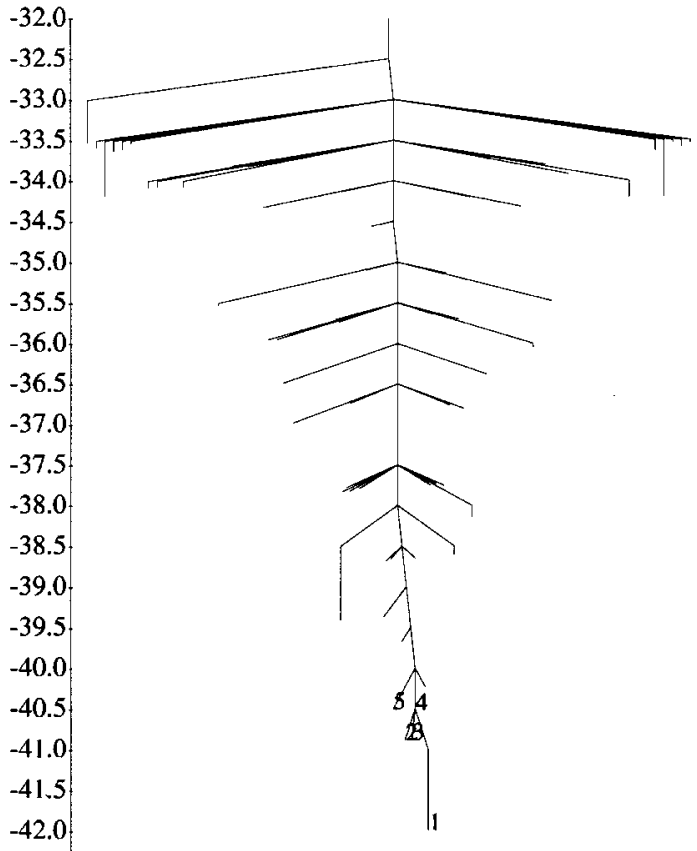

(d)

FIG. 9. Disconnectivity graph for $X_{7} Y_{3}(\sigma, \epsilon)$ values demonstrating that we can control barriers for the selected inherent structures. The energy scale is in units of $\epsilon_{X X}$. Only branches leading to the 70 lowest-energy minima are shown.

\section{ACKNOWLEDGMENTS}

The authors acknowledge support from the National Science Foundation through Award Nos. CHE-0095053 and CHE-0131114. They would also like to thank Dr. M. Miller for helpful discussions and for his gracious assistance with respect to the preparation of the disconnectivity graphs in the present paper.

${ }^{1}$ W. H. Press, S. A. Teukolsky, W. T. Vetterling, and B. P. Flannery, Nu- 
merical Recipes, 2nd ed. (Cambridge University Press, Cambridge, 1992).

${ }^{2}$ S. Kirkpatrick, S. D. Gelatt, Jr., and M. P. Vecchi, Science 220, 4598 (1983).

${ }^{3}$ E. O. Purismia and H. A. Scheraga, Proc. Natl. Acad. Sci. U.S.A. 83, 2782 (1986).

${ }^{4}$ Z. Li and H. A. Scheraga, Proc. Natl. Acad. Sci. U.S.A. 84, 6611 (1987).

${ }^{5}$ D. B. Faken, A. F. Voter, D. L. Freeman, and J. D. Doll, J. Phys. Chem. A 103, 9521 (1999).

${ }^{6}$ Y. H. Lee and B. J. Berne, J. Phys. Chem. A 104, 86 (2000).

${ }^{7}$ D. J. Wales and J. P. K. Doye, J. Phys. Chem. A 101, 5111 (1997).

${ }^{8}$ D. J. Wales and H. A. Scheraga, Science 285, 1368 (1999).

${ }^{9}$ F. H. Stillinger and T. A. Weber, Phys. Rev. A 28, 2408 (1983).

${ }^{10}$ F. H. Stillinger and T. A. Weber, Science 225, 983 (1984).

${ }^{11}$ R. Czerminski and R. Elber, J. Chem. Phys. 92, 5580 (1990).

${ }^{12}$ O. M. Becker and M. Karplus, J. Chem. Phys. 106, 1495 (1997).

${ }^{13}$ M. M. Miller, J. P. K. Doye, and D. J. Wales, J. Chem. Phys. 110, 328 (1999).

${ }^{14}$ J. P. K. Doye, M. M. Miller, and D. J. Wales, J. Chem. Phys. 110, 6896 (1999).

${ }^{15}$ J. P. K. Doye, M. M. Miller, and D. J. Wales, J. Chem. Phys. 111, 8417 (1999).

${ }^{16}$ D. D. Frantz, D. L. Freeman, and J. D. Doll, J. Chem. Phys. 97, 5713 (1992).

${ }^{17}$ C. J. Tsai and K. D. Jordan, J. Chem. Phys. 99, 6957 (1993).

${ }^{18}$ E. Marinari and G. Parisi, Europhys. Lett. 19, 451 (1992).

${ }^{19}$ C. H. Geyer and E. A. Thompson, J. Am. Stat. Assoc. 90, 909 (1995).

${ }^{20}$ J. P. Neirotti, F. Calvo, D. L. Freeman, and J. D. Doll, J. Chem. Phys. 112, 10340 (2000).
${ }^{21}$ F. Calvo, J. P. Neirotti, D. L. Freeman, and J. D. Doll, J. Chem. Phys. 112, 10350 (2000).

${ }^{22}$ J. Jellinek, T. L. Beck, and R. S. Berry, J. Chem. Phys. 84, 2783 (1986).

${ }^{23}$ D. J. Wales and R. S. Berry, J. Chem. Phys. 92, 4283 (1990).

${ }^{24}$ R. S. Berry, Chem. Rev. 93, 2379 (1993).

${ }^{25}$ R. S. Berry, J. Phys. Chem. 98, 6910 (1994).

${ }^{26}$ L. J. Munro, A. Tharrington, and K. D. Jordan, Comput. Phys. Commun. 145, 1 (2002).

${ }^{27}$ N. Tsai and F. F. Abraham, Surf. Sci. 77, 465 (1978).

${ }^{28}$ D. D. Frantz, J. Chem. Phys. 105, 10030 (1996).

${ }^{29}$ D. D. Frantz, J. Chem. Phys. 107, 1992 (1997).

${ }^{30}$ P. Serra and S. Kais, Chem. Phys. Lett. 275, 211 (1997).

${ }^{31}$ Theory of Atomic and Molecular Clusters, edited by J. Jellinek (Springer, Berlin, 1999).

${ }^{32}$ D. L. Freeman and J. D. Doll, Annu. Rev. Phys. Chem. 47, 43 (1996).

${ }^{33}$ M. B. Knickelbein, Annu. Rev. Phys. Chem. 50, 79 (1999).

${ }^{34}$ J. O. Hirschfelder, C. F. Curtiss, and R. B. Bird, Molecular Theory of Gases and Liquids (Wiley, New York, 1954).

${ }^{35}$ C. J. Tsai and K. D. Jordan, J. Phys. Chem. 97, 11227 (1993).

${ }^{36}$ C. J. Cerjan and W. H. Miller, J. Chem. Phys. 75, 2800 (1981).

${ }^{37}$ J. Simons, P. Jorgensen, H. Taylor, and J. Ozment, J. Phys. Chem. 87, 2745 (1983).

${ }^{38}$ A. Banerjee, N. Adams, J. Simons, and R. Shepard, J. Phys. Chem. 89, 52 (1985).

${ }^{39}$ J. Nichols, H. Taylor, P. Schmidt, and J. Simons, J. Chem. Phys. 92, 340 (1990).

${ }^{40}$ D. J. Wales, J. Chem. Phys. 101, 3750 (1994). 
The Journal of Chemical Physics is copyrighted by the American Institute of Physics (AIP). Redistribution of journal material is subject to the AIP online journal license and/or AIP copyright. For more information, see http:/ojps.aip.org/jcpo/jcpcr/jsp Copyright of Journal of Chemical Physics is the property of American Institute of Physics and its content may not be copied or emailed to multiple sites or posted to a listserv without the copyright holder's express written permission. However, users may print, download, or email articles for individual use. 
The Journal of Chemical Physics is copyrighted by the American Institute of Physics (AIP). Redistribution of journal material is subject to the AIP online journal license and/or AIP copyright. For more information, see http://ojps.aip.org/jcpo/jcper/jsp 